\title{
Some BBP-type series for polylog integrals
}

\author{
Anthony Sofo
}

\begin{abstract}
An investigation into a family of definite integrals containing log-polylog functions will be undertaken in this paper. It will be shown that Euler sums play an important part in the solution of these integrals and may be represented as a BBP-type formula. In a special case we prove that the corresponding log integral can be represented as a linear combination of the product of zeta functions and the Dirichlet beta function.
\end{abstract}

\section{Introduction, preliminaries and notation}

In a 1997 paper [2], Bailey, Borwein and Plouffe evaluated various mathematical constants in the form of, for example,

$$
\pi=\sum_{n=0}^{\infty} \frac{1}{2^{4 n}}\left(\frac{4}{8 n+1}-\frac{2}{8 n+4}-\frac{1}{8 n+5}-\frac{1}{8 n+6}\right) .
$$

These types of representations are known as BBP-type series and many other papers have recently been published [1], [20], [22], [23] extending and generalizing various aspects of BBP type series. In general, for a mathematical constant $K$ a BBP-type formula has the form

$$
K=\sum_{n=0}^{\infty} \frac{1}{\alpha^{n}} \sum_{j=0}^{k} \frac{\beta_{j}}{(n k+j)^{p}},
$$

where $\alpha, k, p$ are integers, the base, length and degree of the BBP-type formula, and $\beta_{j}$ are rational numbers. In [5], Coffey gives the representation

$4 G+\frac{3}{4} \zeta(2)-\frac{\pi}{2} \ln 2=\sum_{n=0}^{\infty} \frac{1}{2^{4 n}}\left(\frac{4}{(8 n+1)^{2}}-\frac{2}{(8 n+4)^{2}}-\frac{1}{(8 n+5)^{2}}-\frac{1}{(8 n+6)^{2}}\right)$,

Received June 10, 2021.

2020 Mathematics Subject Classification. Primary 11M06, 11M35, 26B15; secondary 33B15, 42A70, 65B10.

Key words and phrases. Definite integral, BBP-type relations, Euler sums, Dirichlet beta functions.

https://doi.org/10.12697/ACUTM.2021.25.21 
and Adegoke [1] gives the nice representation

$$
\begin{aligned}
& \frac{140}{9} \zeta(3)-\frac{16}{3} \zeta(2) \ln 2 \\
= & \sum_{n=0}^{\infty} \frac{1}{2^{5 n}}\left(\frac{16}{(6 n+1)^{3}}-\frac{24}{(6 n+2)^{3}}-\frac{8}{(6 n+3)^{3}}-\frac{6}{(6 n+4)^{3}}+\frac{1}{(6 n+5)^{3}}\right) .
\end{aligned}
$$

On the example of massless QED, Kim et al. [10] study a skewed Sudokov regime, that is, an asymptotic of the vertex when only one of the two virtualities of the external fermions is sent to zero. In solving an evolution equation for the form factor $S_{k}(z)$, Kim et al derive the BBP type equation

$$
k ! S_{k}(z)=\mathrm{Li}_{k}(z)+\sum_{n=2}^{\infty} z^{n} \sum_{j=1}^{k-1} \frac{1}{n^{k-j}}\left(\begin{array}{c}
k \\
j
\end{array}\right)\left(H_{n-1}\right)^{j}
$$

with harmonic numbers $\left(H_{n-1}\right)^{j}$. In this paper we investigate a family of integrals with polylogarithmic integrand containing some parameters. It will be shown that the solution of these families of integrals may be expressed as a BBP-type representation containing harmonic numbers and including some classical constants such as the Riemann zeta function and the Dirichlet beta function. In particular we investigate a family of integrals of the type

$$
I(a, p, q, t)=\int_{x} \frac{x^{a} \ln ^{p}(x)}{1+x^{2}} \operatorname{Li}_{t}\left(x^{4 q}\right) d x,
$$

where $a \geq-2, p \in \mathbb{N}_{0}, q \in \mathbb{N}, t \in \mathbb{N}_{0}$, for the domain of $x \in(0,1)$. We also study the integral

$$
J(p, q, t)=I(0, p, q, t)=\int_{x} \frac{\ln ^{p}(x)}{1+x^{2}} \operatorname{Li}_{t}\left(x^{4 q}\right) d x
$$

in the positive half line $x \geq 0$. Some other related papers dealing with Euler sums are [3], [12], [18], [13] and the excellent books [19] and [21]. The following special functions will be used in the analysis of the integral (1). The polylogarithm function $\operatorname{Li}_{t}(z)$ is, for $|z| \leq 1$

$$
\operatorname{Li}_{t}(z)=\sum_{m=1}^{\infty} \frac{z^{m}}{m^{t}}
$$

The classical Hurwitz zeta function

$$
\zeta(p, a)=\sum_{n \geq 0} \frac{1}{(n+a)^{p}}
$$

for $\operatorname{Re}(p)>1$ and by analytic continuation to other values of $p \neq 1$, where any term of the form $(n+a)=0$ is excluded. The well known result

$$
\zeta(z)+\eta(z)=2 \lambda(z)
$$


connects the zeta function $\zeta(z)=\sum_{n=1}^{\infty} \frac{1}{n^{z}}$ with the alternating zeta function $\eta(z)$ and the odd zeta function $\lambda(z)$. The zeta function has a simple pole at $z=1$. The Dirichlet beta function $\beta(z)$ or the Dirichlet $L$ function is given by (see Finch [8])

$$
\beta(z)=\sum_{n=0}^{\infty} \frac{(-1)^{n}}{(2 n+1)^{z}} ; z>0,
$$

where $\beta(2)=G$ is the Catalan's constant. The Dirichlet beta function can be represented in powers of $\pi$ at positive odd integer values of $z$, such that

$$
\beta(2 m+1)=\frac{(-1)^{m} E(2 m)}{2^{2 m+2}(2 m) !} \pi^{2 m+1},
$$

where $E(\cdot)$ are the Euler numbers generated by

$$
\operatorname{sech}(z)=\frac{2 e^{z}}{e^{2 z}+1}=\sum_{n=0}^{\infty} \frac{E(n) z^{n}}{n !} .
$$

The Dirichlet beta function which can be extended analytically to the whole complex plane has no singularities in the complex plane and is given by the functional equation

$$
\beta(1-z)=\left(\frac{2}{\pi}\right)^{z} \sin \left(\frac{\pi z}{2}\right) \Gamma(z) \beta(z) .
$$

For complex values of $z, z \in \mathbb{C} \backslash\{0,-1,-2,-3, \ldots\}, \psi(z)$ is the digamma (or psi) function defined by

$$
\psi(z):=\frac{d}{d z}\{\log \Gamma(z)\}=\frac{\Gamma^{\prime}(z)}{\Gamma(z)} .
$$

We know that for $n \geq 1, \psi(n+1)-\psi(1)=H_{n}$ with $\psi(1)=-\gamma$, where $\gamma$ is the Euler-Mascheroni constant and $\psi(n)$ is the digamma function. The polygamma function

$$
\psi^{(k)}(z)=\frac{d^{k}}{d z^{k}}\{\psi(z)\}=(-1)^{k+1} k ! \sum_{r=0}^{\infty} \frac{1}{(r+z)^{k+1}}
$$

and has the recurrence

$$
\psi^{(k)}(z+1)=\psi^{(k)}(z)+\frac{(-1)^{k} k !}{z^{k+1}} .
$$

The connection of the polygamma function with harmonic numbers is,

$$
\begin{aligned}
H_{z}^{(m+1)} & =\zeta(m+1)+\frac{(-1)^{m}}{m !} \psi^{(m)}(z+1), z \neq\{-1,-2,-3, \ldots\} . \\
& =\frac{(-1)^{m}}{m !} \int_{0}^{1} \frac{\left(1-t^{z}\right)}{1-t} \ln ^{m} t d z .
\end{aligned}
$$


The multiplication formula for the polygamma function is

$$
\psi^{(p)}(m z)=\delta_{m, 0} \ln (m)+\frac{1}{m^{p+1}} \sum_{j=0}^{p-1} \psi^{(p)}\left(z+\frac{j}{m}\right),
$$

where $m \in \mathbb{N}$ and $\delta_{m, 0}$ is the Kronecker delta. We expect that integrals of type (1) may be represented by Euler sums and therefore in terms of special functions such as the Riemann zeta function. In a search of the current literature we found some examples for the representation BBP and Euler type sums, see [23]. The papers [16], [14], and [15] also examined some integrals in terms of Euler sums. Some examples will be given highlighting specific cases of the integrals, some of which are not amenable to a computer mathematical package.

\section{Analysis of integrals}

Theorem 1. Let $p, q, t \in \mathbb{N}_{0}$ and $a \geq-2$. Then

$$
\begin{aligned}
I(a, p, q, t)= & \int_{0}^{1} \frac{x^{a} \ln ^{p}(x)}{1+x^{2}} \operatorname{Li}_{t}\left(x^{4 q}\right) d x=\int_{0}^{\frac{\pi}{4}}(\tan \theta)^{a} \ln ^{p}(\tan \theta) \operatorname{Li}_{t}\left(\tan ^{4 q} \theta\right) d \theta \\
& =(-1)^{p} p ! \sum_{n \geq 1} H_{n}^{(t)} \sum_{j=1}^{2 q} \frac{(-1)^{j+1}}{(4 q n+2 j+a-1)^{p+1}},
\end{aligned}
$$

where $H_{n}^{(t)}$ are harmonic numbers of order $t$.

Proof. For $x \in(0,1)$, from (3) and a Taylor series expansion

$$
\operatorname{Li}_{t}\left(x^{4 q}\right)=\sum_{n \geq 1} \frac{x^{4 q n}}{n^{t}}, \frac{1}{1+x^{2}}=\sum_{n \geq 0}(-1)^{n} x^{2 n} .
$$

It is known that the Cauchy product of two convergent series, see Bromwich and Watson [4], is

$$
\left(\sum_{n \geq 0} a_{n} x^{n}\right)\left(\sum_{n \geq 0} b_{n} x^{n}\right)=\sum_{n \geq 0} c_{n} x^{n},
$$

where $c_{n}=\sum_{j=0}^{n} a_{j} b_{n-j}$. It follows that

$$
\frac{x^{a} \mathrm{Li}_{t}\left(x^{4 q}\right)}{1+x^{2}}=\sum_{n \geq 1} H_{n}^{(t)} \sum_{j=1}^{2 q}(-1)^{j+1} x^{4 q n+2 j+a-2}
$$


and therefore

$$
\frac{x^{a} \ln ^{p}(x) \mathrm{Li}_{t}\left(x^{4 q}\right)}{1+x^{2}}=\sum_{n \geq 1} H_{n}^{(t)} \sum_{j=1}^{2 q}(-1)^{j+1} x^{4 q n+2 j+a-2} \ln ^{p}(x) .
$$

Integrating both sides for $x \in(0,1)$, we have

$$
\begin{gathered}
\int_{0}^{1} \frac{x^{a} \ln ^{p}(x) \mathrm{Li}_{t}\left(x^{4 q}\right)}{1+x^{2}} d x=\sum_{n \geq 1} H_{n}^{(t)} \sum_{j=0}^{2 q}(-1)^{j+1} \int_{0}^{1} x^{4 q n+2 j+a-2} \ln ^{p}(x) d x \\
=(-1)^{p} p ! \sum_{n \geq 1} H_{n}^{(t)} \sum_{j=1}^{2 q} \frac{(-1)^{j+1}}{(4 q n+2 j+a-1)^{p+1}}
\end{gathered}
$$

and this is a BBP-type representation for the integral (7) containing harmonic numbers of order $t$. The second integral in (7) is obtained by the substitution $x=\tan \theta$. (7).

The next corollary deals with an alternative representation for the integral

Corollary 1. If $p, t \in \mathbb{N}_{0}, a \geq-2, q>0$ and $a, q \in \mathbb{R}$ then

$$
\begin{gathered}
I(a, p, q, t)=\int_{0}^{1} \frac{x^{a} \ln ^{p}(x)}{1+x^{2}} \operatorname{Li}_{t}\left(x^{4 q}\right) d x \\
=\frac{(-1)^{p} p !}{2^{2 p+2}} \sum_{n \geq 1} \frac{1}{n^{t}}\left(H_{n q+\frac{a-1}{4}}^{(p+1)}-H_{n q+\frac{a-3}{4}}^{(p+1)}\right),
\end{gathered}
$$

where $H_{n q+\frac{a-1}{4}}^{(p+1)}$ are shifted harmonic numbers of order $p+1$.

Proof. A Taylor series expansion of

$$
\operatorname{Li}_{t}\left(x^{4 q}\right)=\sum_{n \geq 1} \frac{x^{4 q n}}{n^{t}} \text { and } \frac{1}{1+x^{2}}=\sum_{j \geq 0}(-1)^{j} x^{2 j}
$$

allows us to write

$$
\begin{aligned}
& I(a, p, q, t)=\sum_{n \geq 1} \frac{1}{n^{t}} \sum_{j \geq 0}(-1)^{j} \int_{0}^{1} x^{4 q n+2 j+a} \ln ^{p}(x) d x \\
&=(-1)^{p} p ! \sum_{n \geq 1} \frac{1}{n^{t}} \sum_{j \geq 0} \frac{(-1)^{j}}{(4 q n+2 j+a+1)^{p+1}} \\
&=\frac{(-1)^{p} p !}{2^{2 p+2}} \sum_{n \geq 1} \frac{1}{n^{t}}\left(\zeta\left(p+1, \frac{1}{4}(4 q n+a+1)\right)-\zeta\left(p+1, \frac{1}{4}(4 q n+a+3)\right)\right)
\end{aligned}
$$




$$
=\frac{(-1)^{p} p !}{2^{2 p+2}} \sum_{n \geq 1} \frac{(-1)^{p}}{p ! n^{t}}\left(\psi^{(p)}\left(q n+\frac{a+3}{4}\right)-\psi^{(p)}\left(q n+\frac{a+1}{4}\right)\right) .
$$

From the relation (5) we obtain the required identity

$$
I(a, p, q, t)=\frac{(-1)^{p} p !}{2^{2 p+2}} \sum_{n \geq 1} \frac{1}{n^{t}}\left(H_{n q+\frac{a-1}{4}}^{(p+1)}-H_{n q+\frac{a-3}{4}}^{(p+1)}\right) .
$$

Remark 1. For $p, q \in \mathbb{N}_{0}$, we see from (8) and (9) the remarkable Euler sum identity

$$
\begin{aligned}
& \sum_{n \geq 1} H_{n}^{(t)} \sum_{j=1}^{2 q} \frac{(-1)^{j+1}}{(4 q n+2 j+a-1)^{p+1}} \\
= & \frac{1}{2^{2 p+2}} \sum_{n \geq 1} \frac{1}{n^{t}}\left(H_{n q+\frac{a-1}{4}}^{(p+1)}-H_{n q+\frac{a-3}{4}}^{(p+1)}\right) .
\end{aligned}
$$

The next corollary deals with some significant special cases of the integral (7).

Remark 2. For the special case $q=1$ we have

$$
\begin{aligned}
I(a, p, 1, t) & =\int_{0}^{1} \frac{x^{a} \ln ^{p}(x)}{1+x^{2}} \operatorname{Li}_{t}\left(x^{4}\right) d x=\frac{(-1)^{p} p !}{2^{2 p+2}} \sum_{n \geq 1} \frac{1}{n^{t}}\left(H_{n+\frac{a-1}{4}}^{(p+1)}-H_{n+\frac{a-3}{4}}^{(p+1)}\right) \\
& =(-1)^{p} p ! \sum_{n \geq 1} H_{n}^{(t)}\left(\frac{1}{(4 n+a+1)^{p+1}}-\frac{1}{(4 n+a+3)^{p+1}}\right)
\end{aligned}
$$

and if we choose $a$ as an odd integer, say $a=1$, we can obtain the representation

$$
I(1, p, 1, t)=\frac{(-1)^{p} p !}{2^{2 p+2}} \sum_{n \geq 1} \frac{1}{n^{t}}\left(H_{n}^{(p+1)}-H_{n-\frac{1}{2}}^{(p+1)}\right)
$$

and using the multiplication formula (6) we have the simplification

$$
I(1, p, 1, t)=\frac{(-1)^{p} p !}{2^{2 p+1}} \sum_{n \geq 1} \frac{1}{n^{t}}\left(H_{n}^{(p+1)}-2^{p} H_{2 n}^{(p+1)}\right)+\frac{(-1)^{p} p !}{2^{p+1}} \zeta(t) \eta(p+1) .
$$

From (11), with $a=1$ and (12) we have for $t \in \mathbb{N}, t \geq 2, p \in \mathbb{N}_{0}$,

$$
\zeta(t) \eta(p+1)=\sum_{n \geq 1} H_{n}^{(t)}\left(\frac{1}{(2 n+1)^{p+1}}-\frac{1}{(2 n+2)^{p+1}}\right)
$$




$$
-\frac{1}{2^{p}} \sum_{n \geq 1} \frac{1}{n^{t}}\left(H_{n}^{(p+1)}-2^{p} H_{2 n}^{(p+1)}\right)
$$

when $t=3, p=2$ we obtain a new representation for the square of Apery's constant

$$
\zeta^{2}(3)=\frac{4}{3} \sum_{n \geq 1} H_{n}^{(3)}\left(\frac{1}{(2 n+1)^{3}}-\frac{1}{(2 n+2)^{3}}\right)-\frac{1}{3} \sum_{n \geq 1} \frac{1}{n^{3}}\left(H_{n}^{(3)}-4 H_{2 n}^{(3)}\right),
$$

and rearranging we obtain the BBP-type representation

$$
5 \zeta(6)+3 \zeta^{2}(3)=\frac{8}{3} \sum_{n \geq 1} H_{n}^{(3)}\left(\frac{1}{(2 n+2)^{3}}-\frac{1}{(2 n+1)^{3}}+\frac{32(-1)^{n+1}}{(2 n)^{3}}\right) .
$$

If we choose $t=5, p=4$ we obtain

$$
\zeta(10)+63 \zeta^{2}(5)=64 \sum_{n \geq 1} \frac{H_{n}^{(5)}}{(2 n+1)^{5}}+64 \sum_{n \geq 1} \frac{H_{2 n}^{(5)}}{n^{5}} .
$$

Similarly for the case $a=1, q=\frac{1}{2}$ we have

$$
I\left(1, p, \frac{1}{2}, t\right)=\frac{(-1)^{p} p !}{2^{p+1}} \zeta(t) \eta(p+1)+\frac{(-1)^{p} p !}{2^{2 p+1}} \sum_{n \geq 1} \frac{1}{n^{t}}\left(H_{\frac{n}{2}}^{(p+1)}-2^{p} H_{n}^{(p+1)}\right) .
$$

In the case $p+1=t, t \in \mathbb{N}, t \geq 2, a=1, q=1$, from (10) we have, after simplification, the new results

$$
\sum_{n \geq 1}\left(\frac{H_{n}^{(t)}}{(2 n+1)^{t}}+\frac{H_{2 n}^{(t)}}{n^{t}}\right)=\frac{1}{2^{t+1}} \zeta(2 t)+\frac{3}{2^{t+1}} \zeta^{2}(t)+\eta(t) \zeta(t)
$$

and

$$
\begin{aligned}
\sum_{n \geq 1}\left(\frac{H_{n}^{(t)}}{(2 n+1)^{t}}-2^{t-1} \frac{(-1)^{n+1} H_{n}^{(t)}}{n^{t}}\right)= & \left(\frac{1-2^{2 t-1}}{2^{t+1}}\right) \zeta(2 t) \\
& +\left(\frac{3-2^{2 t-1}}{2^{t+1}}\right) \zeta^{2}(t)+\eta(t) \zeta(t) .
\end{aligned}
$$

In the next corollary we give two more special cases.

Corollary 2. If $p \in \mathbb{N}, t=1, a \geq-2$ and $q \in \mathbb{N}$ then

$$
\begin{aligned}
I(a, p, q, 1) & =\int_{0}^{1} \frac{x^{a} \ln ^{p}(x)}{1+x^{2}} \ln \left(1-x^{4 q}\right) d x \\
& =(-1)^{p+1} p ! \sum_{n \geq 1} H_{n} \sum_{j=1}^{2 q} \frac{(-1)^{j+1}}{(4 q n+2 j+a-1)^{p+1}}
\end{aligned}
$$


If $p \in \mathbb{N}, t=0, a \geq-2$ and $q \in \mathbb{N}$ then

$$
\begin{gathered}
I(a, p, q, 0)=\int_{0}^{1} \frac{x^{a+4 q} \ln ^{p}(x)}{\left(1+x^{2}\right)\left(1-x^{4 q}\right)} d x \\
=\sum_{j=1}^{2 q}(-1)^{j+1}\left(\frac{p \psi^{(p-1)}\left(\frac{2 j+a-1}{4 q}+1\right)}{q^{p+1} 2^{2 p+2}}+\frac{(2 j+a-1) \psi^{(p)}\left(\frac{2 j+a-1}{4 q}+1\right)}{q^{p+2} 2^{2 p+4}}\right) .
\end{gathered}
$$

Proof. For the case $t=1$, we notice that $\operatorname{Li}_{1}\left(x^{4 q}\right)=-\ln \left(1-x^{4 q}\right)$ and (13) follows from (8). For the case $t=0$, we notice that $\operatorname{Li}_{0}\left(x^{4 q}\right)=\frac{x^{4 q}}{\left(1-x^{4 q}\right)}$. A Taylor series expansion produces

$$
\frac{1}{\left(1+x^{2}\right)\left(1-x^{4 q}\right)}=\sum_{n \geq 1} n \sum_{j=1}^{2 q}(-1)^{j+1} x^{4 q(n-1)+2(j-1)}
$$

in which case

$$
I(a, p, q, 0)=(-1)^{p} p ! \sum_{j=1}^{2 q}(-1)^{j+1} \sum_{n \geq 1} \frac{n}{(4 q n+2 j+a-1)^{p+1}} .
$$

A partial fraction decomposition and simplification leads to (14).

Some examples will follow.

Example 1. We have

$$
\begin{gathered}
I(1,5,2,0)=\frac{59505}{65536} \zeta(6)-\frac{15}{32} \beta(6)-\frac{225}{512} \zeta(5), \\
I(1,4,3,0)=\frac{7}{64} \zeta(4)+\frac{65}{46656 \sqrt{3}} \pi^{5}-\frac{7289}{20736} \zeta(5), \\
I(1,0,1,3)=2 L(3)-\frac{15}{8} \zeta(4)+\frac{1}{2} \zeta(3) \ln 2,
\end{gathered}
$$

and

$$
\begin{aligned}
I(1,4,2,1) & =\frac{29763}{8192} \zeta(6)-\frac{3}{2} \beta(4) G-\frac{2277}{4096} \zeta^{2}(3)-\frac{4371}{2048} \zeta(5) \ln 2 \\
& =24 \sum_{n \geq 1} H_{n}\left(\frac{1}{(8 n+2)^{5}}-\frac{1}{(8 n+4)^{5}}+\frac{1}{(8 n+6)^{5}}-\frac{1}{(8 n+8)^{5}}\right),
\end{aligned}
$$

where $G=\sum_{n \geq 0} \frac{(-1)^{n+1}}{(2 n+1)^{2}}$ is Catalan's constant, $\beta(\cdot)$ is the Dirichlet beta function and where, from the work of Flajolet and Salvy [9],

$L(3)=\sum_{n \geq 1} \frac{(-1)^{n+1} H_{n}}{n^{3}}=\frac{11}{4} \zeta(4)-\frac{7}{4} \zeta(3) \ln 2+\frac{1}{2} \zeta(2) \ln ^{2} 2-\frac{1}{12} \ln ^{4} 2-2 L i_{4}\left(\frac{1}{2}\right)$, 


$$
\begin{aligned}
I(1,2,1,4) & =\frac{3}{16} \zeta(3) \zeta(4)+\frac{137}{8} \zeta(2) \zeta(5)-\frac{1869}{64} \zeta(7) \\
& =\int_{0}^{\frac{\pi}{4}}(\tan \theta) \ln ^{2}(\tan \theta) \operatorname{Li}_{4}\left(\tan ^{4} \theta\right) d \theta \\
I\left(1,2, \frac{1}{2}, 4\right) & =\frac{3}{16} \zeta(3) \zeta(4)+\frac{75}{32} \zeta(2) \zeta(5)-\frac{2141}{512} \zeta(7) \\
I(0,0,1,2) & =\int_{0}^{1} \frac{\operatorname{Li}_{2}\left(x^{4}\right)}{1+x^{2}} d x=2 \sum_{n \geq 1} \frac{H_{n}^{(2)}}{(4 n+1)(4 n+3)} \\
& =\frac{1}{4} \sum_{n \geq 1} \frac{1}{n^{2}}\left(H_{n-\frac{1}{4}}-H_{n-\frac{3}{4}}\right) \\
=12 G \ln 2-\frac{31 \pi^{3}}{48}-\frac{3 \pi}{4} & \ln ^{2} 2-i\left(24 \operatorname{Li}_{3}\left(\frac{1+i}{2}\right)+\frac{5 \pi^{2}}{8} \ln 2-\frac{1}{2} \ln ^{3} 2-\frac{105}{8} \zeta(3)\right) .
\end{aligned}
$$

From Lewin ([11], pp. 164, 296) we have that

$$
\operatorname{Re}\left(\operatorname{Li}_{3}\left(\frac{1+i}{2}\right)\right)=\frac{1}{48} \ln ^{3} 2+\frac{35}{64} \zeta(3)-\frac{5 \pi^{2}}{192} \ln 2
$$

and therefore

$$
I(0,0,1,2)=24 \operatorname{Im}\left(\operatorname{Li}_{3}\left(\frac{1+i}{2}\right)\right)+12 G \ln 2-\frac{31 \pi^{3}}{48}-\frac{3 \pi}{4} \ln ^{2} 2 .
$$

Sofo and Nimbran [17] have shown that the imaginary part of the trilogarithm is

$$
\begin{aligned}
W(3): & =\operatorname{Im}\left(\operatorname{Li}_{3}\left(\frac{1 \pm i}{2}\right)\right)=\sum_{n \geq 1} \frac{\sin \left(\frac{n \pi}{4}\right)}{2^{\frac{n}{2}} n^{3}} \\
& =\sum_{n \geq 1} \frac{(-1)^{n+1}}{2^{2 n}}\left(\frac{2}{(4 n-3)^{3}}+\frac{2}{(4 n-2)^{3}}+\frac{1}{(4 n-1)^{3}}\right),
\end{aligned}
$$

and finally we have

$$
\begin{aligned}
& I(0,0,1,2)=24 W(3)+12 G \ln 2-\frac{31 \pi^{3}}{48}-\frac{3 \pi}{4} \ln ^{2} 2 \\
& I(1,2,2,1)=\int_{0}^{1} \frac{x \ln (x) \operatorname{Li}_{1}\left(x^{8}\right)}{1+x^{2}} d x=\frac{337}{512} \zeta(4)-\frac{1}{4} G^{2}-\frac{77}{128} \zeta(3) \ln 2 \\
&= 2 \sum_{n \geq 1} H_{n}\left(\frac{1}{(8 n+2)^{3}}-\frac{1}{(8 n+4)^{3}}+\frac{1}{(8 n+6)^{3}}-\frac{1}{(8 n+8)^{3}}\right),
\end{aligned}
$$


$I(1,4,3,0)=\int_{0}^{1} \frac{x \ln ^{4}(x) \operatorname{Li}_{0}\left(x^{12}\right)}{1+x^{2}} d x=\frac{7}{64} \zeta(4)+\frac{65}{46656 \sqrt{3}} \pi^{5}-\frac{7289}{20736} \zeta(5)$.

In the next theorem we consider the integral (7) on the positive half line $x \geq 0$.

Theorem 2. For $p, t \in \mathbb{N}$ and $q>0$,

$$
\begin{gathered}
J(p, q, t)=\int_{0}^{\infty} \frac{\ln ^{p}(x) \operatorname{Li}_{t}\left(x^{4 q}\right)}{1+x^{2}} d x=\int_{0}^{\infty} f(x ; p, q, t) d x \\
=\int_{0}^{\frac{\pi}{2}} \ln ^{p}(\tan \theta) \operatorname{Li}_{t}\left(\tan ^{4 q} \theta\right) d \theta \\
=\left(1+(-1)^{p+t+1}\right) I(0, p, q, t) \\
+(-1)^{p+t+1} \frac{(2 \pi i)^{t}}{t !} \int_{0}^{1} \frac{\ln ^{p}(x)}{1+x^{2}} B\left(t, \frac{\ln \left(x^{4 q}\right)}{2 \pi i}\right) d x
\end{gathered}
$$

where

$$
f(x ; p, q, t)=\frac{\ln ^{p}(x)}{1+x^{2}} \operatorname{Li}_{t}\left(x^{4 q}\right),
$$

$I(0, p, q, t)$ is given by (8) or (9) and $B\left(t, \frac{\ln \left(x^{4 q}\right)}{2 \pi i}\right)$ is the Bernoulli polynomial.

Proof. We begin with

$$
J(p, q, t)=\int_{0}^{\infty} \frac{\ln ^{p}(x) \operatorname{Li}_{t}\left(x^{4 q}\right)}{1+x^{2}} d x=\int_{0}^{\infty} f(x ; p, q, t) d x
$$

and put

$$
J(p, q, t)=\int_{0}^{\infty} f(x ; p, q, t) d x=\int_{0}^{1} f(x ; p, q, t) d x+\int_{1}^{\infty} f(x ; p, q, t) d x .
$$

We notice that $f(x ; p, q, t)$ is continuous, bounded and differentiable on the interval $x \in(0,1]$, with $\lim _{x \rightarrow 0^{+}} f(x ; p, q, t)=\lim _{x \rightarrow 1} f(x ; p, q, t)=0$. Now we make the transformation $x y=1$ in the third integral so that

$$
\int_{0}^{\infty} f(x ; p, q, t) d x=\int_{0}^{1} f(x ; p, q, t) d x+(-1)^{p} \int_{0}^{1} \frac{\ln ^{p}(y)}{1+y^{2}} \operatorname{Li}_{t}\left(y^{-4 q}\right) d y .
$$


From Erdélyi et. al. [7], Jonquiěre's relation states

$$
\operatorname{Li}_{s}(z)+e^{i \pi s} \operatorname{Li}_{s}\left(\frac{1}{z}\right)=\frac{\left(2 \pi e^{i \pi}\right)^{s}}{\Gamma(s)} \zeta\left(1-s, \frac{\ln z}{2 \pi i}\right),
$$

where $\operatorname{Li}_{s}(z)$ is a polylogarithm, $\Gamma(s)$ is the Gamma function, $s \in \mathbb{C}$, $\zeta\left(1-s, \frac{\ln z}{2 \pi i}\right)$ is the Hurwitz zeta function and $z$ is not a member of the real interval $[0,1]$. A modified version of (19) is given by Crandall [6] as follows. For an integer $t$ and $z \in \mathbb{C}$,

$$
\operatorname{Li}_{t}(z)+(-1)^{t} \operatorname{Li}_{t}\left(\frac{1}{z}\right)=-\frac{(2 \pi i)^{t}}{t !} B\left(t, \frac{\ln (z)}{2 \pi i}\right)-2 \pi i \Theta(z) \frac{\ln ^{t-1}(z)}{(t-1) !},
$$

where $B\left(t, \frac{\ln (z)}{2 \pi i}\right)$ is the Bernoulli polynomial and $\Theta(z)$ is a time dependent step function

$$
\Theta(z)=\left\{\begin{array}{l}
1, \text { if } \operatorname{Im}(z)<0 \text { or } \mathrm{z} \in[1, \infty), \\
0, \text { otherwise. }
\end{array}\right.
$$

The function $\Theta(z)$ is intended to provide the conventional behavior in the branch when and only when $z$ is in the lower half plane union with the real cut $[1, \infty)$. For convenience we list

$$
\begin{aligned}
& B\left(2, \frac{\ln (z)}{2 \pi i}\right)=\frac{1}{6}+\frac{i}{2 \pi} \ln z-\frac{1}{4 \pi^{2}} \ln ^{2} z \\
& B\left(3, \frac{\ln (z)}{2 \pi i}\right)=-\frac{i}{4 \pi} \ln z+\frac{3}{8 \pi^{2}} \ln ^{2} z+\frac{i}{8 \pi^{3}} \ln ^{3} z .
\end{aligned}
$$

Now we can substitute (20) into (18), so that

$$
\begin{aligned}
\int_{0}^{\infty} f(x ; p, q, t) d x= & \left(1+(-1)^{p+t+1}\right) \int_{0}^{1} f(x ; p, q, t) d x \\
& +(-1)^{p+t+1} \frac{(2 \pi i)^{t}}{t !} \int_{0}^{1} \frac{\ln ^{p}(x)}{1+x^{2}} B\left(t, \frac{\ln \left(x^{4 q}\right)}{2 \pi i}\right) d x .
\end{aligned}
$$

The integral

$$
I(0, p, q, t)=\int_{0}^{1} \frac{\ln ^{p}(x) \operatorname{Li}_{t}\left(x^{4 q}\right)}{1+x^{2}} d x
$$

has been evaluated in Theorem 1 and therefore

$$
\begin{aligned}
J(p, q, t)= & \left(1+(-1)^{p+t+1}\right) I(0, p, q, t) \\
& +(-1)^{p+t+1} \frac{(2 \pi i)^{t}}{t !} \int_{0}^{1} \frac{\ln ^{p}(x)}{1+x^{2}} B\left(t, \frac{\ln \left(x^{4 q}\right)}{2 \pi i}\right) d x
\end{aligned}
$$


and the proof is finished. Note that the integral $I(0, p, q, t)$ does not contribute to $J(p, q, t)$ in the case when $p+t+1$ is an odd integer. The third integral in (15) is obtained by the substitution $x=\tan \theta$.

Remark 3. It can be noted, from Jonquiěre's relation (20) that we are able to determine the value of the integral

$$
\begin{gathered}
\int_{0}^{1} \frac{\ln ^{p}(x) \operatorname{Li}_{t}\left(x^{-4 q}\right)}{1+x^{2}} d x=(-1)^{t+1} I(0, p, q, t) \\
+(-1)^{t+1} \frac{(2 \pi i)^{t}}{t !} \int_{0}^{1} \frac{\ln ^{p}(x)}{1+x^{2}} B\left(t, \frac{\ln \left(x^{4 q}\right)}{2 \pi i}\right) d x .
\end{gathered}
$$

Some examples will follow.

Example 2. If $(p, q, t)=(1,3,3)$ then

$$
\begin{aligned}
J(1,3,3) & =\int_{0}^{\infty} \frac{\ln (x) \operatorname{Li}_{3}\left(x^{12}\right)}{1+x^{2}} d x=\int_{0}^{\frac{\pi}{2}} \ln (\tan \theta) \operatorname{Li}_{3}\left(\tan ^{12} \theta\right) d \theta \\
& =-\frac{(2 \pi i)^{3}}{3 !} \int_{0}^{1} \frac{\ln (x)}{1+x^{2}} B\left(t, \frac{\ln \left(x^{4 q}\right)}{2 \pi i}\right) d x \\
& =-\frac{(2 \pi i)^{3}}{3 !} \int_{0}^{1} \frac{\ln (x)}{1+x^{2}}\left(-\frac{i \ln \left(x^{12}\right)}{4 \pi}+\frac{3 \ln ^{2}\left(x^{12}\right)}{8 \pi^{2}}-\frac{i \ln ^{3}\left(x^{12}\right)}{8 \pi^{3}}\right) d x \\
& =-\frac{89 \pi^{5}}{4}-432 i \pi \beta(4) .
\end{aligned}
$$

If $(p, q, t)=(3,1,5)$ then

$$
\begin{aligned}
J(3,1,5) & =\int_{0}^{\infty} \frac{\ln ^{3}(x) \operatorname{Li}_{5}\left(x^{4}\right)}{1+x^{2}} d x=\int_{0}^{\frac{\pi}{2}} \ln ^{3}(\tan \theta) \operatorname{Li}_{5}\left(\tan ^{4} \theta\right) d \theta \\
& =-\frac{171 \pi^{9}}{16}-53760 i \pi \beta(8)
\end{aligned}
$$


If $(p, q, t)=\left(5, \frac{1}{2}, 6\right)$ then

$$
\begin{gathered}
J\left(5, \frac{1}{2}, 6\right)=\int_{0}^{\infty} \frac{\ln ^{5}(x) \operatorname{Li}_{6}\left(x^{2}\right)}{1+x^{2}} d x \\
=-\frac{50521 i \pi^{12}}{15360}-16 \pi^{6} \beta(6)-3360 \pi^{4} \beta(8)-241920 \pi^{2} \beta(10) .
\end{gathered}
$$

This allows us to also calculate, from (16), that

$$
\begin{aligned}
\int_{0}^{1} \frac{\ln ^{5}(x) \operatorname{Li}_{6}\left(x^{2}\right)}{1+x^{2}} d x= & 1774080 \beta(12)-161280 \pi^{2} \beta(10) \\
& -1792 \pi^{4} \beta(8)-\frac{512}{63} \pi^{6} \beta(6),
\end{aligned}
$$

and, from (21), that

$\int_{0}^{1} \frac{\ln ^{5}(x) \operatorname{Li}_{6}\left(x^{-2}\right)}{1+x^{2}} d x=\frac{50521 i \pi^{12}}{15360}+241920 \pi^{2} \beta(10)+3360 \pi^{4} \beta(8)+16 \pi^{6} \beta(6)$.

If $(p, q, t)=(1,1,6)$ then

$$
\begin{aligned}
J(1,1,6) & =\int_{0}^{\infty} \frac{\ln (x) \operatorname{Li}_{6}\left(x^{4}\right)}{1+x^{2}} d x=\int_{0}^{\frac{\pi}{2}} \ln (\tan \theta) \operatorname{Li}_{6}\left(\tan ^{4} \theta\right) d \theta \\
& =-\frac{61 i \pi^{8}}{30}-6096 \pi \zeta(7)-512 \pi^{2} \beta(6)
\end{aligned}
$$

and

$$
\begin{aligned}
\int_{0}^{1} \frac{\ln (x) \operatorname{Li}_{6}\left(x^{4}\right)}{1+x^{2}} d x= & 14336 \beta(8)-\frac{1408}{3} \pi^{2} \beta(6)-\frac{8}{15} \pi^{4} \beta(4) \\
& -\frac{1}{945} \pi^{6} G-3048 \pi \zeta(7) \\
= & \sum_{n \geq 1} H_{n}^{(6)}\left(\frac{1}{(4 n+3)^{2}}-\frac{1}{(4 n+1)^{2}}\right) .
\end{aligned}
$$

Finally we give the example for $(p, q, t)=(1,2,2)$ :

$$
\begin{gathered}
J(1,2,2)=\int_{0}^{\infty} \frac{\ln (x) \mathrm{Li}_{2}\left(x^{8}\right)}{1+x^{2}} d x=-45 i \zeta(4) \\
+\frac{3}{16} \zeta(2)\left((2+\sqrt{2})\left(\psi^{\prime}\left(\frac{7}{8}\right)-\psi^{\prime}\left(\frac{1}{8}\right)\right)+(2-\sqrt{2})\left(\psi^{\prime}\left(\frac{3}{8}\right)-\psi^{\prime}\left(\frac{5}{8}\right)\right)\right)
\end{gathered}
$$




$$
+\frac{\pi}{64}\left((1+\sqrt{2})\left(\psi^{\prime \prime}\left(\frac{1}{8}\right)+\psi^{\prime \prime}\left(\frac{7}{8}\right)\right)+(1-\sqrt{2})\left(\psi^{\prime \prime}\left(\frac{3}{8}\right)+\psi^{\prime \prime}\left(\frac{5}{8}\right)\right)\right) .
$$

Now, using (16), we have

$$
\begin{gathered}
\int_{0}^{1} \frac{\ln (x) \operatorname{Li}_{2}\left(x^{8}\right)}{1+x^{2}} d x=96 \beta(4)+\frac{135 \sqrt{2}}{4} \zeta(4)-13 \zeta(2) G-7 \pi \zeta(3) \\
\quad+\frac{\pi \sqrt{2}}{64}\left(\psi^{\prime \prime}\left(\frac{1}{8}\right)-\psi^{\prime \prime}\left(\frac{3}{8}\right)\right)-\frac{3 \sqrt{2}}{16} \zeta(2)\left(\psi^{\prime}\left(\frac{1}{8}\right)+\psi^{\prime}\left(\frac{3}{8}\right)\right) \\
=\sum_{n \geq 1} H_{n}^{(2)}\left(\frac{1}{(8 n+7)^{2}}-\frac{1}{(8 n+5)^{2}}+\frac{1}{(8 n+3)^{2}}-\frac{1}{(8 n+1)^{2}}\right) .
\end{gathered}
$$

\section{Concluding Remarks}

We have carried out a systematic study of a family of integrals containing log-polylog functions in terms of Euler sums. We believe that most of our results are new in the literature and we have given many examples, some of which are not amenable to a mathematical computer package.

\section{Acknowledgement}

The author thanks the anonymous referees for constructive comments and suggestions leading to an improvement of this paper.

\section{References}

[1] K. Adegoke, New binary and ternary digit extraction (BBP-type) formulas for trilogarithm constants, New York J. Math. 16 (2010), 361-367.

[2] D. Bailey, P. Borwein, and S. Plouffe, On the rapid computation of various polylogarithmic constants, Math. Comp. 66 (1997), 903-913.

[3] N. Batir, On some combinatorial identities and harmonic sums, Int. J. Number Theory 13 (2017), 1695-1709.

[4] T. J. Bromwich and G. N. Watson, An Introduction to the Theory of Infinite Series, Merchant Books, New York, 2008.

[5] M. W. Coffey, Evaluation of a ln tan integral arising in quantum field theory, J. Math. Phys. 49(9) (2008), 093508, 15 pp.

[6] R. Crandall, Unified algorithms for polylogarithm, L-series, and zeta variants, Algorithmic Reflections: Selected Works, PSIpress, 2012, www.marvinrayburns.com/UniversalTOC25.pdf.

[7] A. Erdélyi, W. Magnus, F. Oberhettinger, and F. G. Tricomi, Higher Transcendental Functions 1, Krieger, New York, 1981.

[8] S. R. Finch, Mathematical constants II, Encyclopedia of Mathematics and its Applications, 169. Cambridge University Press, Cambridge, 2019.

[9] P. Flajolet and B. Salvy, Euler sums and contour integral representations, Experiment. Math. 7 (1998), 15-35.

[10] V. T. Kim, V. A. Matveev, and G. B. Pivovarov, Skewed Sudakov regime, harmonic numbers, and multiple polylogarithms, Phys. Rev. D 99(2) (2019), 025016, 6 pp. 
[11] R. Lewin, Polylogarithms and Associated Functions, North Holland, New York, 1981.

[12] A. Sofo, Integral identities for sums, Math. Commun. 13 (2008), 303-309.

[13] A. Sofo, New classes of harmonic number identities, J. Integer Seq. 15(7) (2012), Article 12.7.4, $12 \mathrm{pp}$.

[14] A. Sofo, Shifted harmonic sums of order two, Commun. Korean Math. Soc. 29 (2014), 239-255.

[15] A. Sofo, General order Euler sums with rational argument, Integral Transforms Spec. Funct. 30 (2019), 978-991.

[16] A. Sofo and D. Cvijović, Extensions of Euler harmonic sums Appl. Anal. Discrete Math. 6 (2012), 317-328.

[17] A. Sofo and A. S. Nimbran, Euler-like sums via powers of log, arctan and arctanh functions, Integral Transforms Spec. Funct. 31 (2020), 966-981.

[18] A. Sofo and H. M. Srivastava, A family of shifted harmonic sums, Ramanujan J. 37 (2015), 89-108.

[19] H. M. Srivastava and J. Choi, Series Associated with the Zeta and Related Functions, Kluwer Academic Publishers, Dordrecht, 2001.

[20] D. Takahashi, On the computation and verification of $\pi$ using BBP-type formulas, Ramanujan J. 51 (2020), 177-186.

[21] C. I. Vălean, (Almost) Impossible Integrals, Sums, and Series, Problem Books in Mathematics, Springer, Cham, 2019.

[22] C. Wei, Several BBP-type formulas for $\pi$, Integral Transforms Spec. Funct. 26 (2015), 315-324.

[23] W. Zhang, New formulae of BBP-type with different moduli, J. Math. Anal. Appl. 398 (2013), 46-60.

College of Engineering and Science, Victoria University, Australia

E-mail address: anthony.sofo@vu.edu.au 